\title{
Telehealth - A Boon in the Time of COVID 19 Outbreak
}

\author{
Tasneem Sajjad Burhani ${ }^{1}$, Waqar M. Naqvi ${ }^{2}$ \\ ${ }^{1}$ Department of Community Health Physiotherapy, Ravi Nair Physiotherapy College, \\ Wardha, Maharashtra, India. ${ }^{2}$ Department of Community Health Physiotherapy, Ravi \\ Nair Physiotherapy College, Wardha, Maharashtra, India.
}

\section{ABSTRACT}

\section{BACKGROUND}

Since December 2019, a novel coronavirus, SARS-CoV-2, has been identified in a group of community acquired pneumonia patients in Wuhan, Hubei Province, China.(1) Within one month of its discovery, this novel coronavirus was rapidly spreading in all regions of China and 25 countries in Asia-Pacific region, Europe, and South America, North America. With the COVID 19 pandemic rapidly spreading in India and the world, it is imperative that the rehabilitation team understands virus's epidemiology quickly at the beginning stages of this certainly long global epidemic. Coronavirus is a novel virus, and it has no known immunity for most of the world population. This is more contagious and lethal than influenza viruses, and effective medication and a vaccine are months away. Our strategy against this is primarily social distancing and interventions to manage infections.

\section{KEY WORDS}

COVID 19, Coronavirus, Pandemic, Telehealth
Corresponding Author:

Waqar M. Naqvi,

Proressor and HOD,

Department of Community Health

Physiotherapy, Ravi Nair Physiotherapy

College, Wardha, Maharashtra, India.

E-mail:waqar.naqvi@dmimsu.edu.in

DOI: $10.14260 / \mathrm{jemds} / 2020 / 454$

How to Cite This Article:

Burhani TS, Naqvi WM. Telehealth- a boon in the time of COVID 19 outbreak. J. Evolution Med. Dent. Sci. 2020;9(29): 20812084, DOI: 10.14260/jemds/2020/454

Submission 25-04-2020,

Peer Review 09-06-2020,

Acceptance 16-06-2020,

Published 20-07-2020.

Copyright (c) 2020 JEMDS. This is an open access article distributed under Creative Commons Attribution License [Attribution 4.0 International (CC BY 4.0)] 


\section{BACKGROUND}

A global pandemic occurs once in a century which brings havoc to the fragile world population. For this reason, many of us have insufficient knowledge with such cases. The latest breakout of an acute respiratory disease associated with coronavirus, named coronavirus disease 19 (Covid-19), carryover of an animal coronavirus to humans that has triggered a global pandemic over the past 3 months.(2) Previous outbreaks including severe acute respiratory syndrome in 2003 and Middle East respiratory syndrome in 2012 were effectively limited to small regions around the world. No part of the world can any longer claim to be completely safe from the coronavirus outbreak that has now spread to 77 countries, infecting over 90,000 people in a span of just three months. It's a well-established fact that one of the most effective strategies to control spread of any communicable disease is to prevent people's exposure to the infected patient.

In case of a fast-spreading epidemic like the present coronavirus outbreak, this becomes non-negotiable. There are basically two ways in which this can be achieved- quarantine and isolation. Though often used interchangeably in media reports, these terms refer to unique situations. Quarantine is the separation of people who are suspected of having being exposed to the infection, while isolation refers to separation of the infected persons (i.e. confirmed cases) from the general population.(3) This not only limits our capacity to diagnose severely ill victims infected with the virus, but it may also prevent asymptomatic individuals coping with major lifethreatening illness, such as stroke, myocardial infarction from seeking timely care that they would get regularly in normal times. ${ }^{(4)}$

At such a time when isolation is one of the first steps used to counter the COVID-19 epidemic, telehealth is becoming a vital tool for secure and successful communication. Telehealth was listed by the World Health Organization among critical services in the policy of "strengthening the Health Systems Response to COVID-19". According to a recent WHO policy, telehealth will be one of the alternative models for clinical services and clinical decision support.

Telehealth can help by allowing moderately ill patients to seek care they need, while reducing their exposure to other severely ill patients.(5) When movement is restricted throughout the world and entire cities are quarantined, affected populations are susceptible to increased stresses of daily life, unexpected economic burdens, communicable and non-communicable diseases, and various mental health sequelae. Consequently, unique and innovative solutions are called for help to address the critical needs of not only those acutely ill with COVID-19 but also all others who may require medical attention but are unable to receive it due to limited access or lack of resources. Under such conditions, telehealth services become a critical asset, with important implications across the entire health-care delivery spectrum. Furthermore, the associated reduction in resource consumption due to the lower need for personal protective equipment can amount to substantial financial savings when considered at national, continental, or global scale.

Once again, the ongoing coronavirus (COVID-19) pandemic reminds us the importance of using telehealth to provide treatment, especially as means to reduce the risk of cross-contamination caused by direct contact. While everyone managing this time with social distancing, distant jobs, shortened office hours, and widespread closures of buildings, so reliable video communications will continue to be essential for helping people stay connected, particularly in healthcare. Healthcare professionals worldwide use video technologies such as Zoom to perform online consultations, diagnose patients remotely, and provide treatment for routine examination and non-life-threatening diseases. Test symptomatic patients remotely before requiring them to visit an ER, clinic, or other test sites. Reduce travel and provide healthcare professionals with cost-effective continued education/training. Video-based telehealth allows for realtime collaboration and interaction with physician, laboratories, and experts, without geographic constraints. The use of telehealth is becoming increasingly popular and has proven successful in the managing a number of other health conditions. Technology can help to continue delivering physiotherapy services during the pandemic.(6)

\section{TELE PHYSIOTHERAPY DURING PANDEMIC}

Tele-physiotherapy uses information and communication technologies to promote patient's recovery within their own homes. This method serves the needs of many elderly patients, especially those living in remote areas. Its essence, the purpose of tele-physiotherapy is to allow medical experts to treat their patients without requiring patients to travel to the clinic.

The use of tele-physiotherapy is precedent. Previous research have demonstrated the suitability and efficacy of the tele-physiotherapy platform for stroke rehabilitation. Most recently, it has been reported to provide effective rehabilitation services for patients with total knee replacement. There are studies which demonstrated the effectiveness of physiotherapy intervention which are telephone based for patients with knee osteoarthritis.(7)

This can be used to treat vestibular conditions. Other than evaluation and modified approaches, we should inform clients and members of the family, understand how the individual communicates with their familiar environment and make suggestions, make that person confident in their home exercise program, assist them with concerns how and where to perform the exercises they were trained in the clinic, progress activity recommendations, evaluate performance on home exercise equipment, and make suggestions for work place environments and ergonomics.

Telehealth is used synchronously in physical therapy with live video and audio services and asynchronously with remote biometric and imaging sessions, as well as remote monitoring. New telehealth services for physiotherapy includes orthopaedics, neurology, geriatrics, women's health, oncology, wound management, paediatrics, cardiac and pulmonary rehabilitation, and home health care.(8) Clearly, telehealth does not provide any physical therapy services which require hands-on treatment. The Screening of patients requiring inoffice treatment is essential for successful telehealth use. Telehealth is only a tool for promoting best practice and care 
in physical therapy and does not duplicate any unwarranted programs. When telehealth services do not boost the quality of treatment or the outcomes of patients, they should not be used.

Telehealth includes a variety of distance- based healthrelated activities conducted using technology for health care, health promotion, health education, health management, or health research purposes. Numerous telehealth benefits have been identified, including enhanced access to services, cost effectiveness, access to education, better health outcomes, quality of care, quality of life, and increased social assistance. ${ }^{(9)}$ Previously telehealth strategies were used in patient care and self-management services, as well as primarily for stroke and other neurological conditions.

\section{Benefits of Tele Health}

- Providing health awareness and preventive services for high-risk groups.

- Enhancing treatment and efficiency in remote areas with minimal internet access.

- Providing one-on-one behavioural and mental health services or in group settings that patients may attend from their homes.

- Promoting comprehensive and rapid collaboration, as well as measuring disaster preparedness and response in real-time.

- Minimize traveling and deliver cost-effective continuing education/ training to healthcare practitioners.

- Switching to telehealth also gives advantage of not stressing about sufficient test kits or N95 masks or any other personal protective equipment.

- Capable of seeing patients, screening for the drivethrough tests available at a local hospital, handling certain acute diseases and managing their chronic conditions, and keeping them out of urgent care or ER.

\section{Support for Population Wellness}

Video has now become a central component of healthcare provision, not just to improve healthcare delivery but also to expand access to the care. Enhanced real-time connectivity that links the entire community - physicians, nurses, patients, administrators, insurance companies, and others promote well-being of the population and provide equitable access to remote and rural communities.

\section{Telehealth Video-Based Innovations Offer Robust Community Wellness Since}

- Accurate care for the suitable patient shall be given at the appropriate time, in the appropriate place.

- Doctors use technology to more accurately diagnose and control disease and provide care.

- All care stakeholders across the ecosystem collaborate and use the information effectively and efficiently.

- Patients are aware and actively involved in their treatment plan.

\section{Challenges and Opportunities in Telehealth}

The Covid-19 rapid spread, and the possibility that health facilities may be cause of contagion, has focused attention on alternative treatment models that minimize face-to-face interactions between clinicians and patients. Particular interest was shown in video consultations which are already being carried as part of national digital health strategies in many countries.

Not all clinical conditions support video consultations. It is definitely suitable for clinicians who self-isolate. Video may be useful for patients consulting on Covid-19 for people with increased distress (for whom a video evaluation may be more helpful than a telephone conversations), those with moderate symptoms indicative of coronavirus and those with more serious symptoms (when a video evaluation may minimize the need to visit a possibly infectious patient). Well patients looking for advice can be directed to a website or phone message that has been recorded. There could be a exchangeoff between remaining at home and taking thorough examination at the clinic for e.g., in vulnerable elderly patients or in patients with immunosuppression.

Despite these barriers and limitations, the patient response has almost universally been one of relief. When we connect with patients, we hear they were scared to come into the clinic and unaware that telehealth was a possibility. Clinicians are also surprised by the scope of care that can be provided remotely through tele health.

\section{Flattening the Curve}

Telehealth would have a direct effect on flattening the demand curve on health systems worldwide, reducing transmission and spreading the occurrence over a longer period time. Almost all telehealth providers have already encountered a increase in demand. Some of the cases for patients using COVID-19 telehealth include self-isolated/ home-isolated patients, patients with mild cases, patients following discharge. To health professionals: physicians with minor symptoms may still communicate with patients remotely, online health workers trainings. Telehealth could be used as a mental healthcare tool for patients, health professionals and used in COVID 19 testing process (e-schedule for clinical and home testing), and by using e-schedule to monitor the waiting time of patients.

\section{CONCLUSIONS}

Prevention and control of COVID-19 spread is a significant priority of government and health care organizations. Yet current public pressure and uncontained spread need programs to further avoid overcrowding and loss of medical supplies and services in the health care system. Telehealth services are ideal for minimizing crowding in hospitals and clinics by triaging low-acuity patients while also preventing more harmful human exposures and facilitating quality care delivery. In recent years, months, and weeks, as state, federal, and international laws and regulations have evolved to allow greater adoption of telehealth systems (especially during this public health crisis). Healthcare providers are also ideally prepared to accept these programs which are being 
introduced. Although some legal, regulatory, and reimbursement issues remain, the COVID-19 outbreak could provide the right impetus for lawmakers and regulatory agencies to promulgate further measures that encourage more widespread adoption of telehealth.

\section{REFERENCES}

[1] Sahin AR, Erdogan A, Agaoglu PM, et al. 2019 novel coronavirus (COVID-19) outbreak: a review of the current literature. Eurasian J Med Oncol 2020;4(1):1-7.

[2] Portnoy J, Waller M, Elliott T. Telemedicine in the Era of COVID-19. J Allergy Clin Immunol Pract 2020;8(5):148991.

[3] Zhou X, Snoswell CL, Harding LE, et al. The role of telehealth in reducing the mental health burden from COVID-19. Telemed J E Health 2020;26(4):377-9.

[4] Huijbregts MPJ, McEwen S, Taylor D. Exploring the feasibility and efficacy of a telehealth stroke self- management programme: a pilot study. Physiother Can 2009;61(4):210-20.

[5] Koh GCH, Hoenig H. How should the rehabilitation community prepare for 2019-nCoV? Arch Phys Med Rehabil 2020;101(6):1068-71.

[6] Greenhalgh T, Wherton J, Shaw S, et al. Video consultations for covid-19. BMJ 2020;368:m998.

[7] Odole AC, Ojo OD. A telephone-based physiotherapy intervention for patients with osteoarthritis of the knee. Int J Telerehabil 2013;5(2):11-20.

[8] Cottrell MA, O'Leary SP, Swete-Kelly P, et al. Agreement between telehealth and in-person assessment of patients with chronic musculoskeletal conditions presenting to an advanced-practice physiotherapy screening clinic. Musculoskelet Sci Pract 2018;38:99-105.

[9] Kairy D, Tousignant M, Leclerc N, et al. The patient's perspective of in-home telerehabilitation physiotherapy services following total knee arthroplasty. Int J Environ Res Public Health 2013;10(9):3998-4011. 